\title{
Efficient Thermodynamic Description of Multi-Component One-Dimensional Bose Gases
}

\author{
Andreas Klümper ${ }^{1}$ and Ovidiu I. Pâţu ${ }^{1,2}$ \\ ${ }^{1}$ Fachbereich C Physik, Bergische Universität Wuppertal, 42097 Wuppertal, Germany \\ ${ }^{2}$ Institute for Space Sciences, Bucharest-Măgurele, R 077125, Romania
}

\begin{abstract}
We present a new method of obtaining nonlinear integral equations characterizing the thermodynamics of one-dimensional multi-component gases interacting via a delta-function potential. In the case of the repulsive two-component Bose gas we obtain a simple system of two non-linear integral equations, allowing for an efficient numerical implementation, in contrast with the infinite number of coupled equations obtained by employing the Thermodynamic Bethe Ansatz. Our technique makes use of the Quantum Transfer Matrix and the fact that, in a certain continuum limit, multi-component gases can be obtained from appropriate anisotropic spin chains.
\end{abstract}

PACS numbers: 05.30.Jp, 02.30.Ik, 05.70.Ce, 51.30.+i

Introduction. Recent advances in magnetic and optical trapping of ultracold quantum gases have opened new possibilities in investigating the physics of strongly interacting particles in one-dimension [1. Coupled with the fact that the strength of the atomic interaction can be controlled using magnetic Fesbach resonances or statedependent dressed potentials 2, these new experiments provide a fertile ground for testing the theoretical predictions obtained from the study of various integrable models solvable by Bethe Ansatz. As a result, we have witnessed the experimental realization of the Lieb-Liniger model [3] using bosonic ${ }^{87} R b$ atoms [4 8 ] and, moreover, the measured thermodynamics 9 is described very well by the Yang-Yang thermodynamics, which was developed in [10, using, what we call nowadays, the Thermodynamic Bethe Ansatz (TBA) [11. In the case of integrable multi-component 1D gases, TBA produces an infinite number of integral equations [11, 12, which makes the extraction of physical information and comparison with prospective experimental data difficult. In this Rapid Communication, we propose a solution to this problem using the Quantum Transfer Matrix [13, 14, and the connection between multi-component gases and anisotropic spin-chains. Our method has the advantage of providing a finite number of non-linear integral equations (NLIE), which are extremely suited for numerical computations, in stark contrast with the TBA result.

Model. We consider a one-dimensional system of $M$ bosons, of equal mass $m=1 / 2$, with $n$ internal degrees of freedom, interacting via a delta-function potential. The many-body Hamiltonian is

$$
\mathcal{H}=-\sum_{i=1}^{M} \frac{\partial^{2}}{\partial x_{i}^{2}}+2 c \sum_{i<j} \delta\left(x_{i}-x_{j}\right)-\sum_{i=1}^{n} \mu_{i} M_{i},
$$

where $c$ is the coupling constant and we consider $\hbar=$ $k_{B}=1$. The first and the second term in the Hamiltonian (1), represent the kinetic and and the interaction energy, while the third is the Zeeman term, where $M_{i}$ is the number of particles in the hyperfine state $|i\rangle$ and $\mu_{i}$ the respective chemical potential. The interaction is attractive for $c<0$ and repulsive for $c>0$. The model is integrable and was solved in [3, 15, 17.

Despite the fact that the model is integrable, computing the thermodynamics is still an incredibly difficult task. The first attempt was done by Yang and Yang [10] for the spinless bosons using the Thermodynamic Bethe Ansatz. TBA has proved to be an extremely useful technique and was used to obtain information about the thermodynamics of various exactly solvable models [11, however, it has one big shortcoming: for a large class of models it produces an infinite number of coupled NLIE. This is also the case for our model, with the exception of the spinless case $(n=1)$. From a very simplified point of view this is due to the fact that the spectrum of the theory contains infinitely many branches. The numerical implementation of this system of equations requires various truncations, which introduce an uncontrollable source of numerical errors and makes the extraction of relevant physical information extremely difficult. Therefore, it is highly desirable to devise a numerically efficient method, which provides a finite number of NLIE.

Fortunately, in the case of lattice models such a method exists. Developed in 13, 14, the Quantum Transfer Matrix (QTM) approach was successfully applied to a large class of integrable spin-chains and even models of strongly correlated electrons. Within this approach, the thermodynamic properties of the model are obtained from the largest eigenvalue of the QTM, which in the thermodynamic limit gives the free energy. Even though, there is no equivalent of the QTM for continuum models, it is well known [18, 19, that a large class of integrable models can be obtained from lattice ones in suitable continuum limits. We say that an integrable model is the continuum limit of a lattice integrable model if, by performing this limit in the Bethe equations and energy spectrum of the lattice model one obtains the Bethe equations and energy spectrum of the continuum model. The natural consequence is that the thermodynamics of the continuum model can be obtained from the thermodynamics of the lattice model, if we take the same limit. This method will be used in this Rapid Communication, 
allowing us to obtain an efficient thermodynamic description of the two-component Bose gas (for the single component case see [20]).

In this publication we study and give results for the Hamiltonian (1) in dimensionless units, however physical units can be restored easily. For particles with mass $m$ and contact interaction strength $g$, see e.g. (1) in [21], the units of temperature, chemical potential, magnetic field, particle density and susceptibility, compressibility, heat capacity, and entropy per length are $T_{0}=\hbar^{2} /\left(2 m a^{2} k_{B}\right)$, $\mu_{0}=h_{0}=\hbar^{2} /\left(2 m a^{2}\right), d_{0}=1 / a, \chi_{0}=\kappa_{0}=2 m a / \hbar^{2}$, $c_{0}=S_{0}=k_{B} / a$. The quantity $a$ is a length scale that can be chosen freely yielding the dimensionless coupling constant $c=m g a / \hbar^{2}$ appearing in (1). In all figures presented in this paper, physical data are shown in the given units and for dimensionless coupling $c=1$ which is realized for any parameter values of $m$ and $g$ with a suitably chosen $a=\hbar^{2} /(m g)$.

Method. The general strategy is the following: first, we identify the spin-chain from which we will obtain in the appropriate continuum limit our model of interest. The next step is the investigation of the thermodynamics of the lattice model using the QTM technique, which will provide a finite number of NLIE. Finally, the desired result is obtained by taking the continuum limit in the equations for the lattice model. Let us make these general considerations more precise. In the case of the repulsive two-component Bose gas, the relevant lattice model is the $U_{q}(\widehat{s l}(3))$ Perk-Schultz spin-chain [22 26], with periodic boundary conditions characterized by the Hamiltonian

$$
\begin{gathered}
H=J \sum_{j=1}^{L}\left(\cos \gamma \sum_{a=1}^{3} e_{a a}^{(j)} e_{a a}^{(j+1)}+\sum_{\substack{a, b=1 \\
a \neq b}}^{3} e_{a b}^{(j)} e_{b a}^{(j+1)}\right. \\
\left.-i \sin \gamma \sum_{\substack{a, b=1 \\
a \neq b}}^{3} \operatorname{sign}(a-b) e_{a a}^{(j)} e_{b b}^{(j+1)}\right)-\sum_{j=1}^{L} \sum_{a=1}^{3} h_{a} e_{a a}^{(j)},
\end{gathered}
$$

where $\gamma \in(0, \pi)$ determines the anisotropy, $\left(q=e^{i \gamma}\right)$, $L$ is the number of lattice sites, $J>0$ fixes the energy scale, $h_{a}$ are chemical potentials and $e_{a b}^{(j)}=I^{\otimes j-1} \otimes$ $e_{a b} \otimes I^{\otimes L-j}$, with $e_{a b}$ the 3 by 3 matrix with elements $\left(e_{a b}\right)_{i j}=\delta_{a i} \delta_{b j}$. This Hamiltonian is the sum of two terms, $H_{b}$ (in the brackets), and the chemical potential term $H_{c}$, which does not break the integrability of the model 24. The $U_{q}(\widehat{s l}(3))$ Perk-Schultz spinchain can be obtained as the fundamental spin model, (see [27]), associated with the trigonometric Perk-Schultz R-matrix [24], $\mathrm{R}(v)=\sum_{a, b, c, d=1}^{3} \mathrm{R}_{b d}^{a c}(v) e_{a b} \otimes e_{c d}$, with $\mathrm{R}_{a a}^{a a}(v)=\frac{\sin (\gamma+v)}{\sin \gamma}, \mathrm{R}_{a b}^{a b}(v)=\frac{\sin v}{\sin \gamma}, \quad(a \neq b), \mathrm{R}_{a b}^{b a}(v)=$ $e^{i \operatorname{sign}(a-b) v},(a \neq b)$, and all the other elements zero. The transfer matrix $\mathrm{T}(v)$ is the $3^{L}$ by $3^{L}$ matrix with elements $\mathrm{T}_{\mathbf{b}}^{\mathbf{a}}(v)=\sum_{\{\mathbf{c}\}} \prod_{j=1}^{L} \mathrm{R}_{c_{j} b_{j}}^{c_{j+1} a_{j}}(v)$, where $\mathbf{a}, \mathbf{b}$, and c are multiple indices, i.e., $\mathbf{a}=\left(a_{1}, \cdots, a_{L}\right)$ and the sum is over all $\mathbf{c}$ with $c_{1}=c_{L+1}$. The $H_{b}$ part of the Hamiltonian (2) can be obtained as the logarithmic derivative of the transfer matrix $H_{b}=\left.J \sin \gamma \frac{\partial \ln \mathrm{T}(v)}{\partial v}\right|_{v=0}$. In order to treat the model at finite temperature we introduce the $3^{N}$ by $3^{N}$ Quantum Transfer Matrix with elements

$$
\begin{aligned}
\left(\mathrm{T}_{Q T M}\right)_{\mathbf{b}}^{\mathbf{a}}(v)=\sum_{\{\mathbf{c}\}} e^{\beta h_{c_{1}}} \prod_{j=1}^{N / 2} \mathrm{R}_{c_{2 j}}^{c_{2 j+1} a_{2 j}}(v+i u) \\
\times \tilde{\mathrm{R}}_{c_{2 j} c_{2 j} a_{2 j-1}}^{c_{2 j}}(i u-v),
\end{aligned}
$$

where $c_{1}=c_{N+1}, \tilde{\mathrm{R}}_{b d}^{a c}(v)=\mathrm{R}_{d a}^{c b}(v), u=-J \beta \sin \gamma / N$, with $N$ the Trotter number and $\beta=1 / T$. The largest eigenvalue of the QTM, from which the free energy of the model can be obtained, has the form: $\Lambda_{Q T M}(v)=$ $\sum_{j=1}^{3} \lambda_{j}(v)$ [28], with

$$
\lambda_{j}(v)=\phi_{-}(v) \phi_{+}(v) \frac{q_{j-1}(v-i \gamma)}{q_{j-1}(v)} \frac{q_{j}(v+i \gamma)}{q_{j}(v)} e^{\beta h_{j}}
$$

where $\phi_{ \pm}(v)=\left(\frac{\sinh (v \pm i u)}{\sin \gamma}\right)^{N / 2}$ and

$$
q_{j}(v)=\left\{\begin{array}{lr}
\phi_{-}(v) & j=0 \\
\prod_{r=1}^{N / 2} \sinh \left(v-v_{r}^{(j)}\right) & j=1,2 \\
\phi_{+}(v) & j=3 .
\end{array}\right.
$$

The two sets of parameters, $\left\{v_{r}^{(j)}\right\}$, are the Bethe roots of the QTM and satisfy the Bethe equations $\lambda_{j}\left(v_{r}^{(j)}\right) / \lambda_{j+1}\left(v_{r}^{(j)}\right)=-1, r=1, \cdots, N / 2$. The Bethe roots are distributed in certain strips of the complex plane which are independent of temperature and Trotter number. This type of specific distribution allows for the definition of auxiliary functions and the use of Cauchy's theorem in deriving NLIE for the largest eigenvalue [14, 29 32. Our results, which in the continuum limit produce Eqs. 3 and 4 , can be understood as the multi-component generalization of the equations presented in 32 .

Main result. In order to obtain the thermodynamics of the two-component Bose gas we have to perform a particular continuum limit in the NLIE obtained for the $U_{q}(\widehat{s l}(3))$ spin-chain. The spin-chain is characterized by the following parameters: lattice constant $\delta$, number of lattice sites $L$, anisotropy $\gamma$, strength of the interaction $J$, and chemical potentials $h_{1}, h_{2}, h_{3}$. The twocomponent Bose gas is obtained by performing the limit $\gamma=\pi-\epsilon, \delta \rightarrow O\left(\epsilon^{2}\right), L \rightarrow O\left(1 / \epsilon^{2}\right), J \rightarrow O\left(1 / \epsilon^{4}\right), h_{1} \rightarrow$ $O\left(1 / \epsilon^{2}\right)$ with $\epsilon \rightarrow 0$. Performing this limit in the Bethe equations and energy spectrum of the $U_{q}(\widehat{s l}(3))$ spinchain, we obtain the Bethe equations and energy spectrum of a two-component Bose gas characterized by the parameters: length $l=L \delta$, coupling constant $c=\epsilon^{2} / \delta$, mass of the particles $m=J \delta^{2}$, and chemical potentials $\mu_{1}=J \epsilon^{2}+h_{2}-h_{1}, \mu_{2}=h_{3}-h_{2}$. In order to simplify the formulae, we are going to consider $J \delta^{2}=1$ (mass of the particles equal to $1 / 2)$ and introduce $\mu=\left(\mu_{1}+\mu_{2}\right) / 2$ and an effective magnetic field $h=\left(\mu_{1}-\mu_{2}\right) / 2$, where $\mu_{1,2}$ are the chemical potentials of the spin up and down particles. We are now ready to state the main result of this 
Rapid Communication. The thermodynamics of the twocomponent repulsive Bose gas is completely characterized by the following system of nonlinear integral equations:

$$
\begin{aligned}
\ln a_{1}(k)= & -\beta\left(k^{2}-\mu-h\right) \\
& +\left[K_{0} * \ln A_{1}\right](k)+\left[K_{1} * \ln A_{2}\right](k+i \epsilon), \\
\ln a_{2}(k)= & -\beta\left(k^{2}-\mu+h\right) \\
& +\left[K_{2} * \ln A_{1}\right](k-i \epsilon)+\left[K_{0} * \ln A_{2}\right](k),
\end{aligned}
$$

where $A_{i}(k)=1+a_{i}(k), K_{0}(k)=2 c /\left(k^{2}+c^{2}\right), K_{1}(k)=$ $c /[k(k+i c)], K_{2}(k)=c /[k(k-i c)]$ and $[f * g](k)=$ $\frac{1}{2 \pi} \int_{-\infty}^{+\infty} f\left(k-k^{\prime}\right) g\left(k^{\prime}\right) d k^{\prime}$. The grandcanonical potential per length is given by

$$
\phi=-\frac{T}{2 \pi} \int_{-\infty}^{+\infty}\left(\ln A_{1}(k)+\ln A_{2}(k)\right) d k
$$

from which all the other thermodynamic quantities can be obtained. Eqs. 3 and 4 are new in the literature, and to our knowledge this is the first example of an efficient thermodynamic description for a multi-component continuum integrable model at all values of the relevant parameters (temperature, chemical potentials and coupling constant).

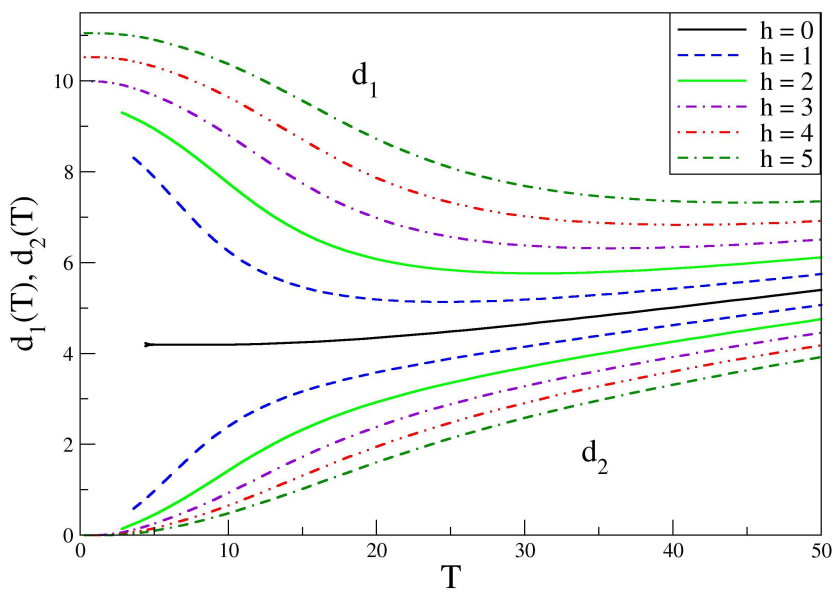

FIG. 1. (Color online) Population densities $d_{1}, d_{2}$ in the upper, lower part of the figure as functions of temperature $T$ for $\mu=15, c=1$ and various effective magnetic fields $h$ (in units of $d_{0}, T_{0}, \mu_{0}$ and $\left.h_{0}\right)$.

Analytic limits. First, we will consider the limit $c \rightarrow 0$. Then, we have $\lim _{c \rightarrow 0} c /\left(k^{2}+c^{2}\right)=\pi \delta(k)$ and $\lim _{c \rightarrow 0} c /[k(k \pm i c)]=0$ and the integral equations decouple

$$
\begin{aligned}
& \ln a_{1}(k)=-\beta\left(k^{2}-\mu-h\right)+\ln \left(1+a_{1}(k)\right), \\
& \ln a_{2}(k)=-\beta\left(k^{2}-\mu+h\right)+\ln \left(1+a_{2}(k)\right) .
\end{aligned}
$$

These equations are easily solvable, and we find

$\phi=\frac{T}{2 \pi} \int_{-\infty}^{+\infty} \ln \left[\left(1-e^{-\beta\left(k^{2}-\mu-h\right)}\right)\left(1-e^{-\beta\left(k^{2}-\mu+h\right)}\right)\right] d k$,

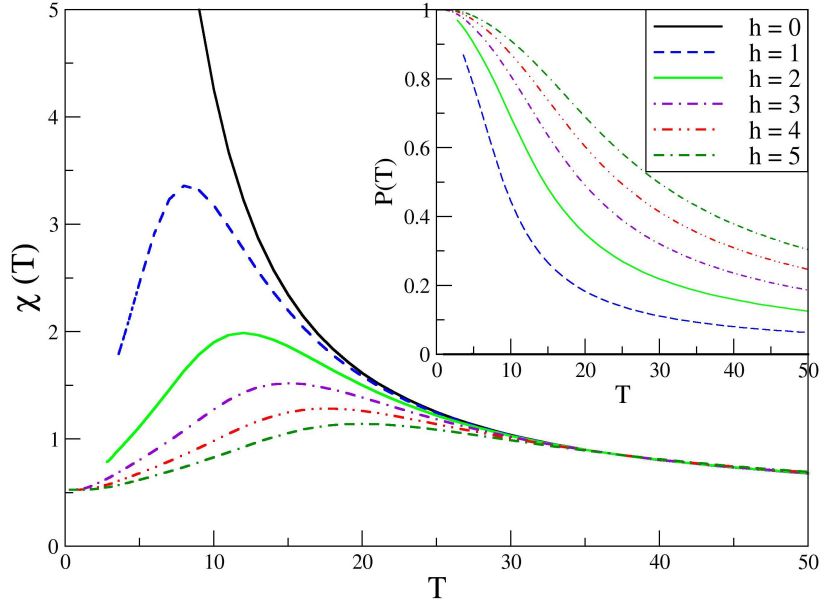

FIG. 2. (Color online) Susceptibility $\chi$ as a function of temperature $T$ for $\mu=15, c=1$ and various effective magnetic fields $h$ (in units of $\chi_{0}, T_{0}, \mu_{0}$ and $h_{0}$ ). Inset: polarization as a function of temperature for the same parameters. In the absence of the magnetic field the polarization is zero.

which is exactly the grandcanonical potential of two noninteracting bosonic gases at different chemical potentials.

In the case of an extremely strong magnetic field, $h \rightarrow$ $\infty$, we expect that the system will become fully polarized and we should obtain the Yang-Yang thermodynamics 10. of the repulsive one-component Bose gas. In this limit $a_{2}(k) \sim 0$ and we obtain

$$
\ln a_{1}(k)=-\beta\left(k^{2}-\mu-h\right)+\left[K_{0} * \ln \left(1+a_{1}\right)\right](k),
$$

and $\phi=-\frac{T}{2 \pi} \int_{-\infty}^{+\infty} \ln \left(1+a_{1}(k)\right) d k$, which is the result obtained in [10]. The same result is obtained in the low temperature limit $(T \ll \mu, h, c)$, when the magnetic field is finite and fixed which shows the ferromagnetic (fully polarized) nature of the groundstate.

As we have mentioned, the numerical implementation of the infinite number of coupled NLIE obtained with the help of TBA [12] is extremely difficult and encounters serious problems in the regime of low magnetic field $(h \ll T, \mu, c)$, or low temperature limit $(0<T \ll h, \mu, c)$. In contrast, Eqs. 3 are easily implementable and provide reliable results in a larger domain of the parameters space. We have checked our results with available numerical data obtained by using the much more involved TBA equations [33] and found perfect agreement. In our case, the regime in which the accuracy is decreasing, is given by the low temperature and low magnetic field limit $(T \rightarrow 0, h \rightarrow 0)$. This is a consequence of a first order phase transition resp. coexistence of fully polarized phases at $T$ and $h$ equal to 0 . It can be seen clearly in Figs. 1 and 2 that, in the absence of the magnetic field the population levels, $d_{i}=-\left(\frac{\partial \phi}{\partial \mu}+(-1)^{i-1} \frac{\partial \phi}{\partial h}\right) / 2, i=1,2$, are equal and, consequently, the polarization defined as $P=\left(d_{1}-d_{2}\right) / d$ with $d=d_{1}+d_{2}$ is zero. In the presence of the magnetic field the groundstate is ferromagnetic, 


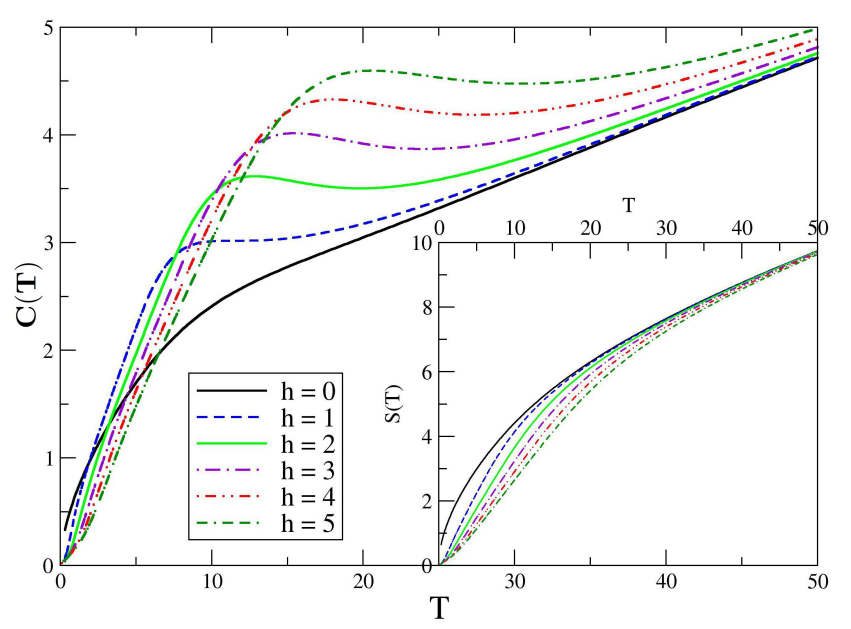

FIG. 3. (Color online) Specific heat $C$ as a function of temperature $T$ for $\mu=15, c=1$ and various effective magnetic fields $h$. Inset: entropy $S=-\frac{\partial \phi}{\partial T}$ as a function of temperature for the same parameters. (All quantities in units of $c_{0}$, $T_{0}, \mu_{0}, h_{0}$ and $\left.S_{0}.\right)$

therefore, the polarization at $T=0$ is +1 , for any positive field ( -1 for negative field) and it decreases at higher temperatures. The ferromagnetic nature of the groundstate, which can also be seen in the $T^{1 / 2}$ behavior of the specific heat $C=T \frac{\partial S}{\partial T}$ at zero magnetic field (see Fig. 3 , is in accordance with a more general theorem of Eisen- berg and Lieb 34] on systems with spin-independent interactions (see also 21). Another consequence of the phase transition is the divergence of the zero-field susceptibility, $\chi=-\frac{\partial^{2} \phi}{\partial h^{2}}$, a feature which can be seen in Fig. 2. The specific heat, magnetic susceptibility, and compresibility, $\kappa=-\frac{\partial^{2} \phi}{\partial \mu^{2}}$, (see Fig. 4), present a nonmonotonic behavior with local maxima shifting to higher temperatures as the magnetic field increases.

In conclusion, we have presented a new method of obtaining a finite number of NLIE characterizing the thermodynamics of integrable multi-component 1D Bose gases, which allows for an efficient numerical treatment and has significant advantages over the TBA result. Fermionic gases can be treated in a similar fashion, but in this case the relevant lattice model for the $n$-component system is the $U_{q}(\widehat{s l}(n \mid 1))$ Perk-Schultz spin-chain. The derivation and a detailed analysis of Eqs. 3 and 4 will be presented in a future publication.

Acknowledgments. The authors would like to thank D.V. Averin, H. Boos, J.S. Caux and F. Göhmann for valuable discussions. The authors are particularly grateful to J.S. Caux for the use of his program for a comparison of the numerical data obtained by the fundamentally different TBA and QTM methods. O.I.P. acknowledges the financial support from the VolkswagenStiftung and the LAPLAS3-PN 0339 program of the Romanian National Authority for Scientific Research.
[1] I. Bloch, J. Dalibard, and W. Zwerger, Rev. Mod. Phys. 80, 885 (2008).

[2] P. Wicke, S. Whitlock, and N.J. van Druten, arXiv: 1010.4545 .

[3] E.H. Lieb and W. Liniger, Phys. Rev. 130, 1605 (1963).

[4] H. Moritz, T. Stöferle, M. Köhl, and T Esslinger, Phys. Rev. Lett. 91, 250402 (2003).

[5] B.L. Tolra et al., Phys. Rev. Lett. 92, 190401 (2004).

[6] B. Paredes et al., Nature 429, 277 (2004).

[7] T. Kinoshita, T. Wenger, and D.S. Weiss, Science 305, 1125 (2004).

[8] L. Pollet, S.M.A. Rombouts, and P.J.H. Denteneer, Phys. Rev. Lett. 93, 210401 (2004).

[9] A.H. van Amerongen et al., Phys. Rev. Lett. 100, 090402 (2008).

[10] C.N. Yang and C.P. Yang, J. Math. Phys. 10, 1115 (1969).

[11] M. Takahashi, Thermodynamics of one-dimensional solvable models, (Cambridge University Press, 1999).

[12] S.J. Gu, Y.Q. Li, Z.J. Ying, and X. A. Zhao, Int. J. Mod. Phys. 16, 2137 (2002).

[13] M. Suzuki, Phys. Rev. B 31, 2957 (1985).

[14] A. Klümper, Ann. Physik 1, 540 (1992).

[15] C.N. Yang, Phys. Rev. Lett. 19, 1312 (1967).

[16] M. Gaudin, Phys. Lett. A 24, 55 (1967).

[17] B. Sutherland, Phys. Rev. Lett. 20, 98 (1968).

[18] P.P. Kulish and E.K. Sklyanin, Phys. Lett. A 70, 461 (1979).
[19] A.G. Izergin and V.E. Korepin, Lett. Math. Phys. 8, 259 (1984).

[20] A. Seel, T. Bhattacharyya, F. Göhmann, and A. Klümper, JSTAT P08030 (2007).

[21] X.W. Guan, M.T. Batchelor, and M. Takahashi, Phys. Rev. A 76, 043617 (2007).

[22] J.H.H Perk and C.H. Schultz, Phys. Lett. A 84, 407 (1981)

[23] O. Babelon, H.J. de Vega, and C.-M. Viallet, Nucl. Physics B 200, 266 (1982).

[24] H.J. de Vega, Int. J. Mod. Phys. A 4, 2371 (1989).

[25] H.J. de Vega and E. Lopes, Phys. Rev. Lett. 67, 489 (1991).

[26] E. Lopes, Nucl. Phys. B 370, 636 (1992).

[27] V.E. Korepin, N.M. Bogoliubov, and A.G. Izergin, Quantum Inverse Scattering Method and Correlation Functions, (Cambridge Univ. Press, 1993).

[28] A. Klümper, T. Wehner, and J. Zittartz, J. Phys. A: Math. Gen. 30, 1897 (1997).

[29] A. Klümper and M. T. Batchelor, J. Phys. A: Math. Gen. 23, L189 (1990).

[30] C. Destri and H. J. de Vega, Phys. Rev. Lett. 69, 2313 (1992).

[31] A. Klümper, Z. Phys. B 91, 507 (1993).

[32] A. Klümper, Lect. Notes Phys. 645, 349 (2004).

[33] J.S. Caux, A. Klauser, and J. van den Brink, Phys. Rev. A 80, 061605 (2009).

[34] E. Eisenberg and E.H. Lieb, Phys. Rev. Lett. 89, 220403 


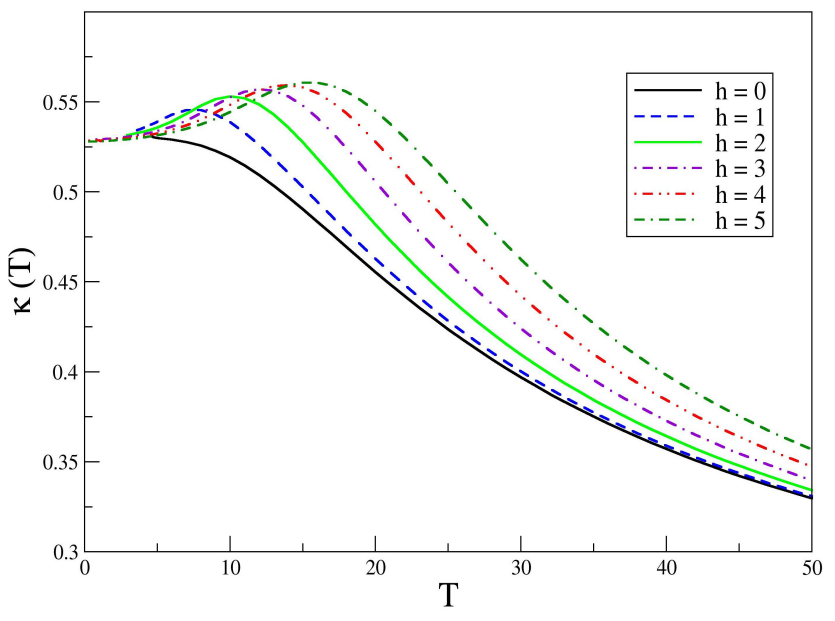

(2002).

FIG. 4. (Color online) Compressibility $\kappa$ as a function of temperature $T$ for $\mu=15, c=1$ and various effective magnetic fields $h$ (in units of $\kappa_{0}, T_{0}, \mu_{0}$ and $h_{0}$ ). 